\title{
Melanoma metastases to the intestines - presentation and management
}

\author{
Michał Aporowicz ${ }^{1}$, Adam Maciejczyk1,2, Rafał Matkowski' ${ }^{1,2}$, Marcin Ziętek¹,2
}

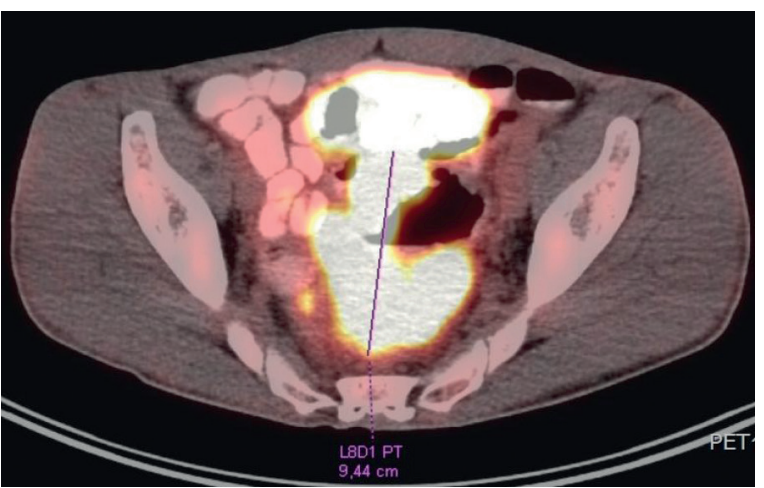

Figure 1. A 49-year-old male with melanoma (pT1NOM1c); a PET scan, a transverse view - a rectosigmoid tumor involving the rectovesica space, $94 \times 71 \times 67 \mathrm{~mm}(\mathrm{AP} \times \mathrm{TR} \times \mathrm{CC})$, standardized uptake value (SUV): 11.1

A 49-year-old male, in follow-up due to melanoma on the back (Clark III, Breslow 0.8 mm - pT1N0), 4.5 years after a re-excision with a sentinel lymph node biopsy, presented with a cramping, epigastric pain, nausea, vomiting, and $10 \mathrm{~kg}$ weight loss. A PET scan revealed a mass in pelvis (fig. 1). The patient underwent sigmoidectomy and resection of infiltrated loops of the small bowel with adjacent mesentery, followed by a stapled side-to-side ileo-ileal and end-to-end colorectal anastomosis. The pathological report confirmed a metastatic melanoma of the small intestine, infiltrating the sigmoid colon and involving mesenteric lymph nodes (8/20; 0/20 mesorectal LN); BRAF(+). The patient received BRAF/MEK inhibitors and anti-PD-L1 immunotherapy (vemurafemib+cobimetinib \pm atezolizumab). Patient has no sign of disease (9 years after first diagnosis, 4 years after laparotomy). Melanoma may metastasize to the lymph nodes, skin, lungs and pleura, brain, liver, bones, adrenal glands, and gastrointestinal tract. Metastases to the small bowel are rare (1-5\%), yet melanoma is the malignancy that most frequently metastasizes to the small intestine (1/3 of all cases). Patients with a newly diagnosed locally advanced melanoma (T4) should undergo an abdominal/pelvic $\mathrm{CT}$ to exclude metastases. Melanoma patients who experience abdominal pain and/or distension, nausea/vomiting, hematochezia/melena should be reevaluated with CT/MRI/PET. Patients with isolated bowel metastases or presenting with bowel obstruction, severe bleeding, perforation should be reffered to surgery with metastasectomy (including regional lymph nodes). Adjuvant systemic therapy is advised, with a regimen depending on a BRAF gene mutation. Despite intestinal metastases, a prolonged survival is possible with appropriate management [1, 2].

This research was financed through statutory subsidies by the Minister of Science and Higher Education as part of the Wrocław Medical University Department of Oncology research grant: SUB. C280.21.023 (record number in the Simple System).

\section{References}

1. Wiśniewski P, Szumera-Ciećkiewicz A, Nasierowska-Guttmejer A. New pathomorphological classification of melanomas. Nowotwory. Journal of Oncology. 2019; 69(3-4): 103-107, doi: 10.5603/njo.2019.0020.

2. Rutkowski P, Wysocki PJ, Nasierowska-Guttmejer A, et al. Cutaneous melanomas. Oncol Clin Pract. 2019; 15(1): 1-19, doi: 10.5603/ OCP.2018.0055.

\section{How to cite:}

Aporowicz M, Maciejczyk A, Matkowski R, Ziętek M. Melanoma metastases to the intestines - presentation and management. NOWOTWORY J Oncol $2022 ; 72: 51$.

This article is available in open access under Creative Common Attribution-Non-Commercial-No Derivatives 4.0 International (CC BY-NC-ND 4.0) license, allowing to download articles and share them with others as long as they credit the authors and the publisher, but without permission to change them in any way or use them commercially. 